\title{
Isolated Mycobacterium Tuberculosis Tenosynovitis and Osteomyelitis of the Foot in an Immunocompetent Patient
}

Elgazzar A ${ }^{1}$, Cecchini $\mathbf{A}^{2}$ and Elmezayen $\mathbf{R I}^{3 *}$ ${ }^{1}$ East Tennessee State University Quillen College of Medicine, USA

${ }^{2}$ Department of Internal Medicine, East Tennessee State University Quillen College of Medicine, USA

${ }^{3}$ Department of Infectious Diseases, Mountain Home Veteran's Affairs Medical Center, USA

*Corresponding author: Elmezayen RI, Department of Infectious Diseases, Mountain Home Veteran's Affairs Medical Center, 809 Lamont St \& Veterans Way, J ohnson City, TN 37604, USA

Received: July 20, 2021; Accepted: August 11, 2021; Published: August 18, 2021

\begin{abstract}
Tubercular osteomyelitis must be considered in the differential diagnosis of immunocompetent patients who present with non-healing wounds with underlying osteomyelitis. Clinical and radiological findings may be indistinguishable from typical bacteria osteomyelitis. A high index of suspicion may prevent delayed diagnosis, and early treatment may prevent subsequent complications. This is a case report of an immunocompetent patient who presented with a chronic non-healing foot wound complicated by abscess and osteomyelitis that did not improve with an appropriate course of intravenous antibiotics. Wound cultures subsequently grew Mycobacterium tuberculosis without evidence of pulmonary involvement. The patient was treated with an initial two-month regimen of rifampin, isoniazid, pyridoxine, pyrazinamide and ethambutol, followed by a tenmonth regimen of rifampin, isoniazid and pyridoxine. The patient experienced significant improvement and complete healing of the foot wound after approximately the first two months of therapy.
\end{abstract}

Keywords: Tuberculosis; Extrapulmonary; Osteomyelitis

\section{Background}

Mycobacterium Tuberculosis (TB) is a common bacterial infection worldwide. It is a major cause of morbidity and mortality, with greater than 10 million new diagnoses and 1.2 million deaths globally in 2018 [1]. Tuberculosis is primarily a lung disease; however, it may affect any organ or tissue, likely via lymphohematogenous spread [2]. Reported cases of extrapulmonary TB include involvement of the lung pleura, lymph nodes, pericardium, bones and joints, as well as the peritoneum and genitourinary tract [2]. Bone and joint involvement of TB in the United States accounts for about $10 \%$ of extrapulmonary cases. While isolated bone infection is very rare, vertebral TB, also known as Pott's disease, is the most common site of skeletal infection. Meanwhile, the foot and ankle are affected in only $0.13 \%$ of extrapulmonary TB cases [3]. Tuberculosis infection is significantly more common in immunocompromised patients, who may develop extrapulmonary manifestations of TB with primary infection or with reactivation of a latent infection.

\section{Case Presentation}

A 72-year-old male Vietnam war veteran with a past medical history of poorly controlled type II diabetes mellitus and chronic kidney disease presented to the hospital with left foot swelling, erythema and a small non-healing wound for 3 months (Figure 1). He was previously treated with outpatient empiric oral antibiotics, but his symptoms failed to improve, and he continued to have the foot erythema and draining wound. Upon hospital presentation, laboratory studies showed WBC of $6.4 \times 10^{3} / \mathrm{uL}$, serum creatinine of $1.5 \mathrm{mg} / \mathrm{dL}$, ESR of $5 \mathrm{~mm} / \mathrm{hr}$, CRP of $4.5 \mathrm{mg} / \mathrm{L}$, and negative blood cultures.

He was admitted and underwent magnetic resonance imaging

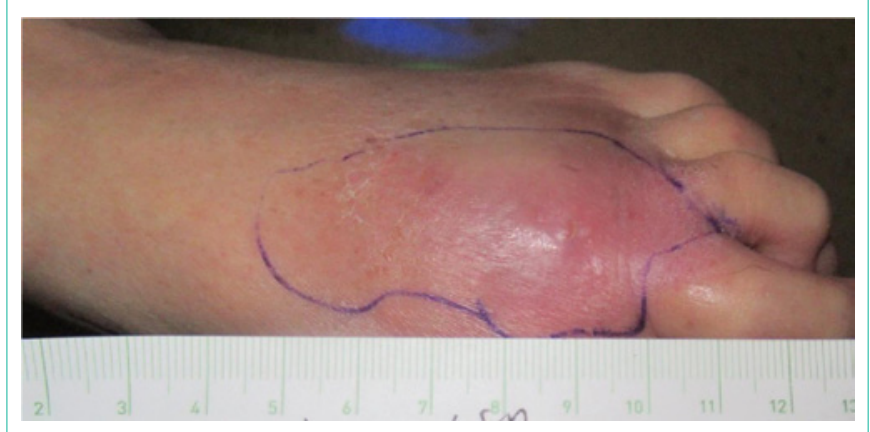

Figure 1: The patient presented with left foot swelling and erythema for 3 months.

(MRI), the results of which revealed subcutaneous abscess, osteomyelitis of the $2^{\text {nd }}$ distal metatarsal and proximal phalanx, as well as septic arthritis of the $2^{\text {nd }}$ Metatarsophalangeal (MTP) joint. He underwent irrigation and debridement of the abscess and ray amputation of the left $2^{\text {nd }}$ metatarsal and toe (Figure 2). The bone was sent for biopsy and cultures. All cultures remained negative, and he was discharged on intravenous ceftriaxone for 6 weeks.

The patient was then transitioned to oral amoxicillin-clavulanic acid due to poor healing.

Approximately 6 weeks after the patient's irrigation and debridement, wound cultures grew Mycobacterium tuberculosis. The wound culture was then sent to National Jewish Health $(\mathrm{NJH})$ in Denver, Colorado where it was confirmed by DNA qPCR as Mycobacterium tuberculosis. The bone biopsy revealed gangrenous necrosis with focally necrotizing granulomas on microscopy and was also confirmed by NJH to be growing Mycobacterium tuberculosis. 


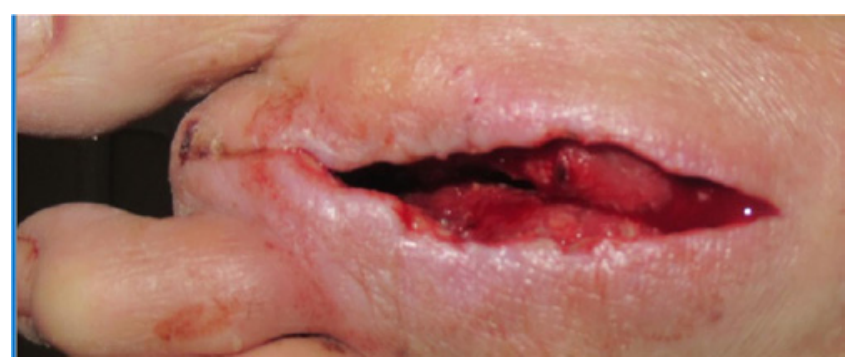

Figure 2: The left foot after irrigation and debridement of the abscess and ray amputation of the left second metatarsal and toe.

Upon further history taking, the patient reported extensive travel history during his military service in Vietnam. However, he did not recall any prior diagnosis or treatment of tuberculosis. He underwent a chest x-ray which revealed no acute cardiopulmonary processes noting absence of focal consolidation or cavitation, pulmonary edema, or pleural effusion. Further workup revealed a nonreactive human immunodeficiency virus (HIV) antibody screen and no other signs of immune deficiency. In addition, the patient did not have any clinical stigmata of tuberculosis, and reported no fever, weight loss, night sweats or cough.

Based on the confirmed MTP osteomyelitis and septic joint, the patient was treated with directly observed therapy consisting of an initial two-month regimen of rifampin, isoniazid, pyridoxine, pyrazinamide and ethambutol, followed by a ten-month regimen of rifampin, isoniazid and pyridoxine. The patient remained compliant with his regimen and experienced significant improvement and complete healing of the foot wound after approximately the first two months of therapy. The patient was followed periodically and completed 12 total months of therapy. He developed mild foot neuropathy for which he was continued on pyridoxine. He was referred to his primary care provider for continued management of his uncontrolled diabetes.

\section{Discussion}

Infection with tuberculosis in the United States is common in patients who have emigrated from endemic areas. Risk factors for TB in the United States include poverty, homelessness, alcohol and substance use, and importantly, immune compromise, most notably in patients with HIV. However, military personnel and veterans who serve in countries endemic for tuberculosis carry a high risk of infection as well. In patients with both Acquired Immunodeficiency Syndrome (AIDS) and tuberculosis, greater than 50\% will have extrapulmonary involvement [4].

Skeletal TB occurs most often in the vertebrae and can lead to destruction of the vertebrae and disc. Patients often present with back pain, but in severe cases, may present with symptoms of cord compression such as paralysis, paresthesia, and loss of bowel or bladder control. Abscesses of the adjacent paraspinal and psoas muscles in vertebral TB osteomyelitis are a known complication and may present with similar findings along with fever and even sepsis [4]. Non vertebral osteomyelitis and tenosynovitis of the foot are very unusual sites of TB infection. Interestingly, our patient had high risk exposure during his military service although trauma to the limb during the military was not reported.
Diagnosing extrapulmonary TB is a challenge and should be done via isolation of Mycobacterium tuberculosis from culture of a bodily fluid or tissue sample. The Center for Disease Control and Prevention (CDC) recommends Acidfast Bacilli (AFB) smear microscopy on all patients suspected of having pulmonary or extrapulmonary TB. However, a negative AFB smear does not rule out pulmonary TB, and false positive results are common. Culture is required to correctly identify the species. Once growth is detected, the sample must be sent to a reference laboratory for definitive speciation and drug susceptibility testing [5]. Our patient's culture was initially sent for routine, anaerobic, fungal and AFB smear microscopy and culture. Upon growth detection in the culture, samples were sent to $\mathrm{NJH}$, where both confirmatory testing and drug susceptibility testing were performed.

The CDC also recommends that histopathologic examination of biopsied specimens be performed. Microscopy of a tissue specimen infected with TB typically reveals granulomatous inflammation. The central portion of granulomas typically have a characteristic caseating necrotic appearance. Our patient's histopathology was reported as gangrenous necrosis with focally necrotizing granulomas.

It is important to rule out pulmonary tuberculosis in cases presenting with extrapulmonary disease. Chest radiograph is an essential component of TB diagnosis; it may reveal various types of infiltrates, fibrosis/scarring, cavitation, lymphadenopathy, a tuberculoma or lung mass, or possibly scattered fibronodular densities in the case of miliary TB [2]. Our patient's chest x-ray did not demonstrate any abnormalities.

Radiographic studies of bone in TB osteomyelitis typically show demineralization with preservation of joints and cartilage in early disease, however it can progress to complete bone destruction in late stages [6]. Radiographic features such as sclerosis or osteolytic lesions may be present but are also seen in conditions such as inflammatory arthritis. The non-specific nature of these findings may lead to an incorrect diagnosis and delay in proper treatment. Thus, MRI is the preferred modality to diagnose osteomyelitis. Findings may include bone and cartilage destruction, soft tissue changes representing abscess, and inflammation with displacement of the surrounding structures [7]. Our patient's MRI demonstrated joint dislocation and bone marrow enhancement compatible with osteomyelitis in addition to multiloculated abscess and infectious tenosynovitis.

Treatment for tuberculosis is culture/sensitivities-driven, especially as multi-drug resistant $\mathrm{TB}$ is becoming more common. Treatment differs between pulmonary and extrapulmonary tuberculosis. Treatment of pulmonary TB consists of 4 drugs for 8 weeks, followed by 2 drugs for 4-7 months, while extrapulmonary TB requires a longer duration of therapy. Our patient completed 12 months of therapy composed of 4 drugs for 8 weeks, followed by 10 months of 2 drugs with complete resolution of his foot infection. According to the CDC/American Thoracic Society treatment guidelines, this is also the treatment regimen for patients with extrapulmonary TB affecting the bone, joint or spine [5]. Our patient underwent this treatment regimen and experienced complete healing.

\section{References}

1. MacNeil A, Glaziou P, Sismanidis C, Date A, Maloney S, Floyd K. Global epidemiology of tuberculosis and progress toward meeting global targets- 
worldwide, 2018. Morbidity and Mortality Weekly Report. 2020; 69: 281.

2. Jameson JL. Harrison's principles of internal medicine. McGraw-Hill Education. 2018

3. Leonard MK, Blumberg HM. Musculoskeletal tuberculosis. Microbiology spectrum. 2017; 5 .

4. Golden MP, Vikram HR. Extrapulmonary tuberculosis: an overview. American family physician. 2005; 72: 1761-1778.

5. Lewinsohn DM, Leonard MK, LoBue PA, Cohn DL, Daley CL, Desmond E, et al. Official American Thoracic Society/Infectious Diseases Society of America/Centers for Disease Control and Prevention clinical practice guidelines: diagnosis of tuberculosis in adults and children. Clinical Infectious Diseases. 2017; 64: e1-e33.

6. Abdelwahab IF, Bianchi S, Martinoli C, Klein M, Hermann G. Atypical extraspinal musculoskeletal tuberculosis in immunocompetent patients, a review. Part I: atypical osteoarticular tuberculosis and tuberculous osteomyelitis. Canadian Association of Radiologists Journal. 2006; 57: 86.

7. Shah J, Patkar D, Parikh B, Parmar H, Varma R, Patankar T, et al. Tuberculosis of the sternum and clavicle: imaging findings in 15 patients. Skeletal radiology. 2000; 29: 447-453. 